\title{
Lipoprotein subclass measurements by nuclear magnetic resonance spectroscopy improve the prediction of coronary artery disease in Type 1 Diabetes. A prospective report from the Pittsburgh Epidemiology of Diabetes Complications Study
}

\author{
S. S. Soedamah-Muthu' ${ }^{2,5}$, Y.-F. Chang ${ }^{2,3}$, J. $\operatorname{Otvos}^{4}$, R. W. Evans ${ }^{2}$, T. J. Orchard ${ }^{1}$ \\ ${ }^{1}$ University of Pittsburgh, Diabetes and Lipid Research Bldg., Pittsburgh, USA \\ 2 Department of Epidemiology, Graduate School of Public Health, University of Pittsburgh, Pittsburgh, PA, USA \\ ${ }^{3}$ Department of Neurosurgery, University of Pittsburgh, Pittsburgh, PA, USA \\ ${ }^{4}$ LipoScience, Raleigh, NC, USA \\ ${ }^{5}$ Department of Epidemiology and Public-Health, Royal Free and University College London Medical School, London, UK
}

\begin{abstract}
Aim/hypothesis. To examine whether nuclear magnetic resonance lipoprotein spectroscopy improves the prediction of coronary artery disease in patients with Type 1 diabetes, independently of conventional lipid and other risk factors.

Methods. A prospective nested case-control design of subjects with childhood onset Type 1 diabetes from the Pittsburgh Epidemiology of Diabetes Complications Study was used. 59 controls were age-, sex- and duration-matched to 59 incident cases of coronary artery disease (fatal or non-fatal myocardial infarction, angina, coronary stenosis $>50 \%$ ) occurring during 10 years of follow-up. Lipid mass and particle concentrations of VLDL, LDL, and HDL subclasses, grouped into three size categories (large, medium, and small), were assessed prior to event with nuclear magnetic resonance spectroscopy.

Results. Univariate analyses showed that both lipid mass and particle concentrations of all three VLDL subclasses, small LDL, medium LDL, and medium
\end{abstract}

HDL were increased in CAD cases compared to controls, while large HDL was decreased. Mean LDL and HDL particle sizes were lower in cases. In multivariate models using conventional lipid and non-lipid risk factors, triglycerides and overt nephropathy were the strongest predictors of CAD. Nuclear magnetic resonance measures further improved the prediction, i.e. large HDL particle concentration $(\mathrm{OR}=0.43$, $p=0.030)$, medium HDL mass $(\mathrm{OR}=3.79, p=0.026)$ and total VLDL particle concentration $(\mathrm{OR}=2.33$, $p=0.033$ ).

Conclusion/interpretation. While these results underscore the importance of triglycerides and overt nephropathy in CAD risk in Type 1 diabetic patients, they also suggest that nuclear magnetic resonance lipoprotein spectroscopy could further refine its prediction and show novel findings concerning HDL subclasses. [Diabetologia (2003) 46:674-682]

Keywords Nuclear magnetic resonance spectroscopy, Type 1 diabetes mellitus, coronary artery disease, lipids, lipoproteins.
Although patients with Type 1 diabetes have a cardiovascular mortality risk which is up to 10 -fold higher compared to non-diabetic subjects [1], conventional

Received: 22 July 2002 / Revised: 12 December 2002

Published online: 13 May 2003

C) Springer-Verlag 2003

Corresponding author: Dr. T. J. Orchard, University of Pittsburgh, Diabetes and Lipid Research Bldg., 3512 Fifth Avenue, Pittsburgh, PA 15213 USA

E-mail: tjo@pitt.edu

Abbreviations: CAD, Coronary artery disease; NMR, Nuclear magnetic resonance; eGDR, estimated glucose disposal rate. lipid or lipoprotein concentrations tend to be relatively normal in Type 1 diabetic patients with adequate glycaemic control [2, 3]. In contrast, Type 1 diabetic patients with poor glycaemic control [4] and those with diabetic nephropathy $[5,6]$ show atherogenic lipid abnormalities consistent with the increased risk of cardiovascular morbidity and mortality which accompanies development of diabetic nephropathy [7].

Thus, an important part of the cardiovascular risk in Type 1 diabetic patients could be mediated by atherogenic lipid abnormalities. However, limited information is available for Type 1 diabetic patients with coronary artery disease (CAD), although increased 
levels of total and LDL cholesterol and triglycerides have been documented [8, 9], as have lipoprotein compositional abnormalities $[10,11]$.

Studies on non-diabetic subjects have shown that measurements of lipoprotein subclass distributions by electrophoresis or ultracentrifugal separation, could enhance assessment of CAD risk, for example, as with the increased risk seen in subjects who have a preponderance of small, dense LDL (pattern B) particles $[12,13,14]$. Specific effects of HDL subclasses on cardiovascular disease have also been observed, ie. a protective effect for large HDL subfractions $\left(\mathrm{HDL}_{2 \mathrm{~b}}\right)$ [15] and an increased risk for small HDL particles $\left(\mathrm{HDL}_{3 \mathrm{~b}}\right.$ and $\left.{ }_{3 \mathrm{c}}\right)[16]$.

Unlike traditional separation methods, which are laborious and time-consuming and usually measure subclass levels in relative (percentage) terms, NMR spectroscopy is automated and provides rapid, direct quantification of several VLDL, LDL, and HDL subclasses simultaneously $[17,18]$. The method relies on the observation that each lipoprotein subclass emits a lipid spectral signal that varies in a characteristic way as a function of particle diameter, irrespective of differences in lipid composition.

To date, only two studies of Type 1 diabetic subjects have used NMR lipoprotein subclass analysis $[19,20]$, and neither examined relations with incident CAD. The aim of our study was therefore to examine whether NMR-measured lipoprotein subclasses improve the prediction of CAD in patients with Type 1 diabetes beyond that achieved using conventional risk factors and chemically-measured lipids. Separate evaluations were conducted using NMR concentrations expressed either in particle number or in lipid mass concentration units.

\section{Subjects and Methods}

The Pittsburgh Epidemiology of Diabetes Complications (EDC) study is a 10-year prospective follow-up study of risk factors for complications in childhood onset Type 1 diabetes mellitus. Full details of the design and recruitment in the EDC study have been published elsewhere [21]. All study participants gave informed consent and the protocol was approved by the relevant ethics committee.

Study design and population. Between 1950-1980, 658 Type 1 diabetic patients were recruited from the Children's Hospital of Pittsburgh (CHP) registry, which has been shown to be representative of the Allegheny County population [22]. All subjects were diagnosed before the age of 17 years and first seen at CHP within a year of diagnosis and were on insulin therapy at discharge. These 658 subjects were first assessed in the period 1986 to 1988 and biennially thereafter, for a total of 10 years follow-up, ending in the period 1996 to 1998. Subjects refusing clinic attendance completed a medical history questionnaire. A nested case-control study was designed using the 10 year follow-up data, selecting 71 CAD cases who had plasma available prior to the event. Of these, four cases had missing risk factor data. A similar number of control subjects who had not developed a coronary event were pair matched by age ( \pm 3 years), sex, and duration ( \pm 3 years) of diabetes. Of these 67 pairs, 59 had (enzymatically assessed) fasting triglyceride levels less than $4.5 \mathrm{mmol} / \mathrm{l}$, permitting LDL cholesterol calculations, which formed the basis of these analyses.

Measurements. Before attending the clinic, all participants completed a questionnaire concerning demographic information and medical history. An ever-smoker was defined as a person who has smoked 100+ lifetime cigarettes. Sitting blood pressure was measured according to the Hypertension Detection and Follow-up Program protocol [23] using a random zero sphygmomanometer. Hypertension was defined as blood pressure greater than or equal to $140 / 90 \mathrm{~mm} \mathrm{Hg}$ or the taking of anti-hypertensive medication.

Laboratory techniques. Fasting blood samples were taken including an EDTA plasma sample separated within $30 \mathrm{~min}$ of blood draw and frozen at $-70^{\circ} \mathrm{C}$ until being sent for NMR spectroscopy and other analyses. Total glycosylated haemoglobin $\left(\mathrm{HbA}_{1}\right)$ values were originally measured using cationexchange microcolumn chromatography (Isolab, Akron, Ohio), up to October 26, 1987 after which high-performance liquid chromatography was used (Diamat, Bio-Rad, Hercules, Calif., USA) (interassay CV of $2.25 \%$ ).

Cholesterol and triglycerides were measured enzymatically $[24,25]$. HDL cholesterol was asayed using a modification of the Lipid Research Clinics method by a heparin and manganese procedure [26]. LDL cholesterol was calculated using the Friedewald equation [27], which has been previously validated in this population [28]. Non-HDL cholesterol was calculated as total cholesterol minus HDL cholesterol. Apolipoprotein A1 and apolipoprotein B were measured by immuno-electrophoresis [29]. Fibrinogen was assayed with a biuret colormetric procedure and a clotting method, and white blood cell (WBC) counts using the Coulter S-Plus IV.

Estimated Glucose Disposal Rate (eGDR), an inverse marker of insulin resistance, was calculated using a previously described regression equation (involving $\mathrm{HbA}_{1}$, WHR and hypertension) derived from hyperinsulinaemic euglycaemic clamp studies [30].

Lipoprotein subclass profiles were measured on freshlythawed frozen plasma samples by proton NMR spectroscopy (LipoScience, Raleigh, N.C., USA) [17, 18, 31]. The NMR method uses the characteristic signals broadcast by lipoprotein subclasses of different size as the basis of their quantification. Each subclass signal emanates from the aggregate number of terminal methyl groups on the lipids contained within the particle. Cholesterol esters and triglycerides in the particle core each contribute three methyl groups and phospholipids and unesterified cholesterol in the surface shell each contribute two methyl groups. To a close approximation, the diameter of the particle determines the number of methyl groups present (and hence, the amplitude of the methyl NMR signal), irrespective of differences in lipid composition arising from, for example, variations in the relative amounts of cholesterol ester and triglycerides in the particle core, varying degrees of unsaturation of the lipid fatty acyl chains, varying phospholipid composition, etc. For this reason, the methyl NMR signal emitted by each subclass serves as a direct measure of the concentration of that subclass.

NMR spectra of each plasma specimen $(0.25 \mathrm{ml})$ were acquired in duplicate at $47^{\circ} \mathrm{C}$ on an automated $400 \mathrm{MHz}$ lipoprotein analyzer (LipoScience) and the lipid methyl signal envelope decomposed computationally to give the amplitudes of the contributing signals of 16 VLDL, LDL, and HDL subclasses. Using calculations based on standard assumptions about relations between lipoprotein diameter and lipid content, 
the NMR spectral data can be transformed to give subclass concentrations expressed either in particle concentration units (nanomoles of particles per litre, nmol/l) or, alternatively, in cholesterol or triglyceride mass concentration units $(\mathrm{mg} / \mathrm{dl}$ or $\mathrm{mmol} / \mathrm{l}$ ). Conversion factors to relate these signal amplitudes to either particle concentration or lipid mass concentration units were obtained from NMR and chemical analysis of a set of purified subclass standards. These reference standards were isolated from a diverse group of normo- and dyslipidemic individuals by a combination of ultracentrifugation and agarose gel filtration chromatography and characterized for size distribution by electron microscopy (VLDL, LDL) or polyacrylamide gradient gel electrophoresis (HDL). Triglyceride and cholesterol measurements on each subclass standard were also carried out. Particle concentrations (nmol/l) were derived for each subclass standard by determining the total concentration of core lipid (cholesterol ester plus triglycerides) and dividing the volume occupied by these lipids by the core volume per particle calculated from knowledge of the particle's diameter [32]. Lipid mass concentrations of VLDL subclasses are expressed in $\mathrm{mmol} / \mathrm{l}$ triglyceride units and those of the LDL and HDL subclasses in $\mathrm{mmol} / \mathrm{l}$ cholesterol units. Summing the relevant subclass concentrations gives NMR-derived values for total VLDL triglycerides, LDL cholesterol, and HDL cholesterol. It is emphasized that these NMR-derived lipid values come from direct measurement of the lipoprotein particles carrying the lipids, not from actual lipid measurements. They are, in effect, the lipid values expected for a person with "normal" lipoprotein particles, since the conversion calculations assume that each subclass particle contains the same, normal amount of cholesterol and triglycerides contained in the corresponding isolated subclass standard.

For data analysis, the 16 measured subclasses were grouped into the following 10 subclass categories: large VLDL (60$200 \mathrm{~nm})$, medium VLDL (35-60 nm), small VLDL (27$35 \mathrm{~nm})$, intermediate-density lipoprotein, or IDL (23-27 nm), large LDL (21.3-23 nm), medium LDL (19.8-21.2 nm), small LDL (18.3-19.7 nm), large HDL (8.8-13 nm), medium HDL $(8.2-8.8 \mathrm{~nm})$, and small HDL (7.3-8.2 nm). Weighted average VLDL, LDL, and HDL particle sizes (nm diameter) were computed as the sum of the diameter of each subclass multiplied by its relative mass percentage as estimated from the amplitude of its methyl NMR signal. LDL and HDL subclass distributions determined by NMR and gradient gel electrophoresis are highly correlated [33, 34]. NMR-derived LDL and IDL subclass diameters are uniformly about $5 \mathrm{~nm}$ smaller because they are referenced to diameters assayed by electron microscopy [35]. There was no evidence of changes in NMR measurement related to the time of storage of the frozen samples.

Complications. Retinopathy was determined by standard 3-field fundus photographs read by the Fundus Photography Reading Center, University of Wisconsin (Madison) and defined as advanced background retinopathy (grade 40-50) and proliferative retinopathy (grade $>60$ ) compared to mild or no retinopathy (grade <30) [36].

Overt nephropathy (ON) was defined as having an albumin excretion rate greater than $200 \mu \mathrm{g} / \mathrm{min}$ in at least two of three timed urine collections (24-h, overnight and 4-h post-clinic), renal failure (serum creatinine $>440 \mu \mathrm{mol} / \mathrm{l}$ ), undergoing dialysis, or a kidney transplantation. Microalbuminuria was defined as an albumin excretion rate between 20 and $200 \mu \mathrm{g} / \mathrm{min}$. Urinary albumin was determined immuno-nephelometrically [37] (interassay coefficient of variation of $15.2 \%$ ). Urinary creatinine concentrations were measured using an analyzer (Ekachem 400 analyzer, Eastman Kodak, Rochester, N.Y., USA) (interassay CV of 5.6\%).
Neuropathy was assessed by vibration threshold determination and included peripheral neuropathy, or distal symmetric polyneuropathy determined according to the Diabetes Control and Complications Trial clinical examination protocol [38, 39].

Clinical CAD was defined using standardized criteria as a history of MI confirmed by ECG Q-waves, Minnesota Codes (MC) 1.1, 1.2, or hospital records, fatal CAD based on death certificates coded according to standard methodology, coronary revascularisation or coronary artery occlusion greater than or equal to $50 \%$ by angiography. Ischaemic ECG was defined as MC 1.3, 4.1 to $4.3,5.1$ to 5.3 , or 7.1. Angina was diagnosed by the EDC physician at each visit.

Statistical analysis. Statistical analyses were carried out using SAS statistical package (SAS, Cary, N.C., USA). Log-transformations were used for variables with skewed distributions. The paired Student's $t$ test was used for conventional, lipid risk factors and NMR-derived lipoprotein measures to test for significant differences between CAD cases and controls. McNemar tests were used for the difference between CAD cases and controls in categorical variables. Spearman rank correlations were used to assess associations between NMR-derived variables and other risk factors. Stepwise conditional logistic regression models were used to assess the order of importance of these new and conventional risk factors. Standardised odds ratios were estimated from these models, $\operatorname{Exp}(\beta * \mathrm{SD})$. The nested models were compared by the Akaike's Information Criterion (AIC):

$\mathrm{AIC}=-2 *[\log (\mathrm{L})-\mathrm{q}]$

$\log (\mathrm{L})$ is the $\log$-likelihood evaluated at the maximum likelihood estimates of the model parameters, and $\mathrm{q}$ is the number of parameters in the model. The first term is a measure of how well the model fits the data, and the second term is a penalty for the addition of parameters. The model giving the smallest value of AIC or log likelihood is selected by the criterion as the best model.

A $p$ value of less than 0.05 was considered statistically significant.

\section{Results}

As expected, age, sex and duration of diabetes (matching variables) were equally distributed between the 59 CAD cases and controls (Table 1). Subjects with subsequent CAD were more likely to be an ever smoker, have overt nephropathy and retinopathy, to have a higher WHR and a lower eGDR. Higher mean non-HDL cholesterol, triglycerides, apolipoprotein $\mathrm{B}$ and $\mathrm{A} 1 / \mathrm{HDL}$ cholesterol ratio and lower mean HDL cholesterol concentrations were found for CAD cases.

Unadjusted relationships of incident CAD with mean levels of NMR-measured lipoprotein subclasses expressed in lipid mass concentration units are shown (Table 2). Similar data for NMR particle number concentration are also shown (Table 3 ). In addition, values for the average VLDL, LDL, and HDL particle sizes in the CAD case and control groups are reported (Table 2). Levels of total VLDL and all three VLDL subclasses were higher for the CAD cases compared to the controls, using either measure. However, mean 
Table 1. Risk factors for incident CAD. Pittsburgh Epidemiology of Diabetes Complications Study. Ten year matched case-control analysis: means $(\mathrm{SD})$ or $\%(n)$

\begin{tabular}{|c|c|c|}
\hline & $\begin{array}{l}\text { No CAD }(n=59) \\
\text { Means }(\mathrm{SD})\end{array}$ & $\begin{array}{l}\text { CAD }(n=59) \\
\text { Means (SD) }\end{array}$ \\
\hline \multicolumn{3}{|l|}{ Non-lipid risk factors } \\
\hline Age (years) & $35.0(6.0)$ & $34.5(6.0)$ \\
\hline Duration (years) & $27.0(6.9)$ & $27.1(7.0)$ \\
\hline Waist-to-hip ratio & $0.81(0.08)$ & $0.83(0.08)^{*}$ \\
\hline $\operatorname{BMI}\left(\mathrm{kg} / \mathrm{m}^{2}\right)$ & $24.5(3.1)$ & $24.0(3.7)$ \\
\hline Estimated glucose disposal rate ( $\mathrm{mg} / \mathrm{kg} / \mathrm{min})$ & $7.78(2.42)$ & $6.85(2.11)^{*}$ \\
\hline Systolic blood pressure $(\mathrm{mm} \mathrm{Hg})$ & $120.0(22.8)$ & $121.5(18.5)$ \\
\hline Diastolic blood pressure (mm Hg) & $72.1(11.6)$ & $74.4(12.0)$ \\
\hline White blood cell count $\left(* 10^{3} / \mathrm{mm}^{2}\right)$ & $6.93(1.78)$ & $7.55(2.27)$ \\
\hline Fibrinogen $(\mu \mathrm{mol} / \mathrm{l})$ & $8.7(2.8)$ & $9.3(2.9)$ \\
\hline $\operatorname{Sex}(\% \operatorname{men}(n))$ & $48(28)$ & $48(28)$ \\
\hline Microalbuminuria $(\%(n))$ & $27(16)$ & $32(19)$ \\
\hline Neuropathy (\% (n)) & $42(25)$ & $44(26)$ \\
\hline \multicolumn{3}{|l|}{ Lipid risk factors } \\
\hline LDL cholesterol (mmol/l) & $2.96(0.78)$ & $3.26(1.03)(\mathrm{p}=0.07)$ \\
\hline HDL cholesterol (mmol/l) & $1.51(0.33)$ & $1.26(0.32) * * *$ \\
\hline Cholesterol $(\mathrm{mmol} / \mathrm{l})$ & $4.87(0.83)$ & $5.17(1.18)^{(\mathrm{p}=0.09)}$ \\
\hline Non-HDL cholesterol (mmol/l) & $3.36(0.89)$ & $3.91(1.22) * *$ \\
\hline Triglycerides $(\mathrm{mmol} / \mathrm{l})^{\#}$ & $0.72(0.51-0.90)$ & $1.17(0.81-1.57) * * *$ \\
\hline Apo B $(\mathrm{g} / \mathrm{l})$ & $0.95(0.19)$ & $1.12(0.31)^{* * *}$ \\
\hline Apo A1/HDL cholesterol & $2.49(0.59)$ & $2.90(0.67) * * *$ \\
\hline
\end{tabular}

${ }^{*} p<0.05 * * p \leq 0.01 * * * p \leq 0.001$, \#Log transformed before statistical testing, median and interquartile range presented

Table 2. Incident CAD by levels of NMR measured lipoprotein subclasses, expressed in lipid mass concentration units, and average lipoprotein particle size

\begin{tabular}{|c|c|c|}
\hline & $\begin{array}{l}\text { No CAD }(n=59) \\
\text { Means }(\mathrm{SD})\end{array}$ & $\begin{array}{l}\text { CAD }(n=59) \\
\text { Means }(\mathrm{SD})\end{array}$ \\
\hline Small VLDL (mmol/l triglyceride) & $0.31(0.16)$ & $0.43(0.18) * * *$ \\
\hline Large VLDL (mmol/l triglyceride) & $0.05(0.13)$ & $0.19(0.30) * * *$ \\
\hline Total VLDL (mmol/l triglyceride) & $0.54(0.34)$ & $0.99(0.61) * * *$ \\
\hline VLDL size $(\mathrm{nm})$ & $39.2(12.6)$ & $41.3(7.0)$ \\
\hline Large LDL (mmol/l cholesterol) & $1.80(0.76)$ & $1.57(1.01)$ \\
\hline Total LDL (mmol/l cholesterol) & $3.01(0.62)$ & $3.31(1.10)^{(\mathrm{p}=0.049)}$ \\
\hline LDL size $(\mathrm{nm})$ & $21.0(0.5)$ & $20.6(0.6) * *$ \\
\hline Small HDL (mmol/l cholesterol) & $0.44(0.16)$ & $0.40(0.16)$ \\
\hline Medium HDL (mmol/l cholesterol) & $0.10(0.15)$ & $0.20(0.20)^{* * *}$ \\
\hline Large HDL (mmol/l cholesterol) & $0.81(0.43)$ & $0.49(0.35) * * *$ \\
\hline
\end{tabular}

$* p<0.05 * * p \leq 0.01 * * * p \leq 0.001$

VLDL size did not differ between the two groups. Among the LDL subclasses, small LDL and medium LDL levels were increased in CAD cases compared to controls, with total LDL expressed as lipid mass being borderline significant $(p=0.049)$, but significant ex- pressed as particle concentration $(p \leq 0.01)$. Average LDL size was also smaller in cases versus controls (20.6 vs $21.0 \mathrm{~nm}$ ).

A higher proportion of detectable levels of IDL subclasses were found in the CAD cases compared to 
Table 3. Incident CAD by levels of NMR-measured lipoprotein subclasses, expressed in particle concentration units

\begin{tabular}{|c|c|c|}
\hline & $\begin{array}{l}\text { No CAD }(n=59) \\
\text { Mean }(\mathrm{SD})\end{array}$ & $\begin{array}{l}\text { CAD }(n=59) \\
\text { Mean }(\mathrm{SD})\end{array}$ \\
\hline Small VLDL (nmol/l) & $65.8(33.4)$ & $96.6(46.6) * * *$ \\
\hline Large VLDL (nmol/l) & $0.6(1.3)$ & $1.9(2.8) * * *$ \\
\hline Total VLDL (nmol/1) & $74.0(35.3)$ & $115.9(48.8) * * *$ \\
\hline Small LDL (nmol/l) & $526(309)$ & $800(545)^{* * *}$ \\
\hline Total LDL (nmol/1) & $1334(284)$ & $1547(535)^{* *}$ \\
\hline Small HDL (nmol/l) & $18176(6275)$ & $16261(6466)^{(\mathrm{p}=0.17)}$ \\
\hline Medium HDL (nmol/l) & $2846(4029)$ & $5556(5364) * * *$ \\
\hline Large HDL (nmol/l) & $8924(4087)$ & $5747(3264) * * *$ \\
\hline Total HDL (nmol/1) & $29946(4589)$ & $27564(4963) * *$ \\
\hline
\end{tabular}

$* p<0.05, * * p \leq 0.01, * * * p \leq 0.001$

Table 4. Spearman correlations of enzymatically-measured lipids and NMR-measured lipoprotein subclasses $(n=118)$

\begin{tabular}{|c|c|c|c|c|}
\hline Small VLDL (mmol/l triglyceride) & $0.44^{* * * *}$ & $0.53^{* * *}$ & $-0.38^{* * *}$ & $0.53^{* * *}$ \\
\hline Medium VLDL (mmol/l triglyceride) & $0.82^{* * *}$ & $0.36^{* * *}$ & $-0.62^{* * *}$ & $0.54^{* * *}$ \\
\hline Large VLDL (mmol/l triglyceride) & $0.68^{* * *}$ & 0.14 & $-0.50^{* * *}$ & $0.34^{* * *}$ \\
\hline Total VLDL (mmol/l triglyceride) & $0.85^{* * *}$ & $0.49^{* * *}$ & $-0.67^{* * *}$ & $0.66^{* * *}$ \\
\hline Small LDL (mmol/l cholesterol) & $0.53^{* * *}$ & $0.37^{* * *}$ & $-0.37^{* * *}$ & $0.49^{* * *}$ \\
\hline Large LDL (mmol/l cholesterol) & $-0.36^{* * *}$ & $0.33^{* * *}$ & $0.42^{* * *}$ & 0.16 \\
\hline Total LDL (mmol/l cholesterol) & $0.19^{*}$ & $0.72^{* * *}$ & -0.06 & $0.69^{* * *}$ \\
\hline Small HDL (mmol/l cholesterol) & -0.13 & $0.22 *$ & -0.11 & 0.13 \\
\hline Medium HDL (mmol/l cholesterol) & $0.30^{* * *}$ & 0.05 & 0.04 & 0.16 \\
\hline Large HDL (mmol/l cholesterol) & $-0.70^{* * *}$ & $-0.36^{* * *}$ & $0.87^{* * *}$ & $-0.50^{* * *}$ \\
\hline Total HDL (mmol/l cholesterol) & $-0.63^{* * *}$ & $-0.28^{* *}$ & $0.88^{* * *}$ & $-0.40^{* * *}$ \\
\hline
\end{tabular}

$* p<0.05, * * p \leq 0.01, * * * p \leq 0.001$

the control group ( $29 \%$ vs $14 \%$, chi-square $p=0.05$, data not shown in table).

Levels of total HDL and large HDL subclass were lower in those with CAD compared to the controls with both measures, while levels of small HDL did not differ. In contrast, levels of the medium-size HDL subclass were found to be higher in those with CAD.

Spearman rank correlations between enzymatically measured lipids and NMR spectroscopy measured lipoprotein subclasses are shown (Table 4). There was a high correlation between enzymatically measured triglycerides and NMR-derived total VLDL $(\mathrm{r}=0.85$, $p \leq 0.001$ ), total LDL cholesterol and NMR-derived total LDL mass $(\mathrm{r}=0.72, p \leq 0.001)$ and between total HDL cholesterol and NMR-derived total HDL mass $(\mathrm{r}=0.88, p \leq 0.001)$. Enzymatically measured triglyceride levels were also correlated with NMR derived medium HDL ( $\mathrm{r}=0.30)$. Enzymatic HDL cholesterol did not correlate with NMR-derived small or medium HDL. Although $\mathrm{HbA}_{1}$ correlated with small VLDL $(\mathrm{r}=0.24, p \leq 0.01)$ and medium LDL $(\mathrm{r}=0.23, p<0.05)$, there were no correlations between $\mathrm{HbA}_{1}$ and other
VLDL, LDL and any of the HDL subclasses (data not shown in Table).

To assess the effect of each set (mass, size, particle number) of NMR variables on the prediction of CHD, all of these were added one by one to a Multivariate conditional logistic regression model with established risk factors (Model 2, Table 5). Models 3 to 5 assessed the independent predictive effect of each NMR set of variables above the established risk factors. Only those risk factors which were related to CHD in univariate models (Table 1, Table 2) were entered in these models in Table 5. The independent predictive effect was assessed, limited by the high correlations between these risk factors.

The most important non-lipid risk factor for CAD was overt nephropathy (Table 5, Model 1). There was no independent relationship with WHR, eGDR, smoking and retinopathy. As only one CAD case was on lipid lowering therapy and none of the subjects had used aspirin, the effect of these drugs could not be assessed. There were however, 26 patients on blood pressure lowering drugs ( $\geq 1$ of the following: ACE in- 
Table 5. Multivariate models for CAD associated with NMR lipoprotein concentrations

\begin{tabular}{|c|c|c|c|c|c|c|}
\hline & Risk factors & $\beta(\mathrm{SE})$ & OR $(95 \% \mathrm{CI})$ & $p$-value & $-2 \log \mathrm{L}$ & AIC \\
\hline Model 1: Non-lipid risk factors & $\mathrm{ON}$ & $1.79(0.62)$ & $6.00(1.77-20.37)$ & 0.004 & 66.75 & 68.75 \\
\hline Model 2: Model $1+$ enzymatic lipid risk factors & $\mathrm{TG}$ & $2.03(0.57)$ & $3.08(1.66-5.72)^{\#}$ & 0.0004 & 55.93 & 57.93 \\
\hline Model 3: Model $2+$ NMR lipoprotein mass levels & VLDLTG & $0.04(0.01)$ & $5.30(1.92-14.64)^{\#}$ & 0.0013 & 43.82 & 47.82 \\
\hline Model 4: Model 2 + NMR lipoprotein particle sizes & $\mathrm{TG}$ & $2.03(0.57)$ & $3.08(1.66-5.72)^{\#}$ & 0.0004 & 55.93 & 57.93 \\
\hline \multirow[t]{2}{*}{ Model 5: Model $2+$ NMR lipoprotein particle levels } & $\mathrm{ON}$ & $2.43(1.04)$ & $11.41(1.48-88.06)$ & 0.020 & \multirow[t]{2}{*}{33.21} & \multirow[t]{2}{*}{41.21} \\
\hline & VLDLpno & $0.02(0.01)$ & $2.36(1.08-5.13)^{\#}$ & 0.031 & & \\
\hline \multirow[t]{4}{*}{ Model 6: Only significant variables from model 1-5 } & $\mathrm{ON}$ & $2.37(1.04)$ & $10.71(1.40-82.07)$ & 0.022 & \multirow[t]{4}{*}{32.87} & \multirow[t]{4}{*}{40.87} \\
\hline & Medium HDL & $0.19(0.09)$ & $3.79(1.17-12.25)^{\#}$ & 0.026 & & \\
\hline & Large HDLpno & $-0.21(0.10)$ & $0.43(0.20-0.92)^{\#}$ & 0.030 & & \\
\hline & VLDLpno & $0.02(0.01)$ & $2.33(1.07-5.08)^{\#}$ & 0.033 & & \\
\hline
\end{tabular}

Risk factors available for modeling were:

Non-lipid risk factors: eGDR, smoking, overt nephropathy $(\mathrm{ON})$, retinopathy, WHR, blood-pressure lowering drugs (yes/no) If $\mathrm{HbA}_{1}$ was used instead of eGDR the model is unchanged.

Lipid risk factors: HDL (cholesterol), TG (log-transformed), Non-HDL, Apo B, Apo A1/HDLc ratio.

NMR lipoprotein lipid mass levels: Large VLDL, Medium VLDL, Small VLDL, Total VLDL (VLDLTG), Small LDL,

hibitors, calcium channel blockers, beta blockers and diuretics), 19 CAD cases and 7 controls (chi-square $p=0.005$ ). The use of blood pressure lowering drugs was entered into model 1 and did not alter the results.

On making enzymatically-measured lipids available to the model, triglycerides replaced overt nephropathy and improved the prediction (Table 5, Model 2). If lipoprotein lipid mass concentrations derived by NMR were made available (Table 5, Model 3), prediction was further improved by the replacement of triglycerides with medium HDL cholesterol mass and total VLDL triglyceride mass. IDL was entered into model 3, but did not add to the prediction of CAD. Use of NMRderived average lipoprotein particle sizes in place of NMR subclass concentrations did not improve the model (Table 5, Model 4). Availability of NMR particle concentration information (Table 5, Model 5) instead of NMR lipid mass concentration information (Table 5, Model 3) resulted in a slightly improved prediction, with large HDL, medium HDL, total VLDL, and overt nephropathy as independent risk factors for CAD. This final model was confirmed in a further analysis (Table 5, Model 6) in which only the significant variables from the previous models were available.

When the data set was expanded to include the further eight matched pairs, in which fasting triglycerides were not available, but non-HDL cholesterol was, similar results were obtained, with VLDL particle concentration $(\mathrm{OR}=3.54,95 \% \mathrm{CI}: 1.63-7.68, p=0.001)$, medium HDL mass $(\mathrm{OR}=3.10$, 95\% CI: 1.34-7.19, $p=0.008)$, and overt nephropathy $(\mathrm{OR}=5.76,95 \% \mathrm{CI}$ : $1.13-29.42, p=0.03$ ), but not large HDL particle concentration, being retained in the model.
Medium LDL, IDL (detectable yes/no), Total LDL, Large HDL, Medium HDL (H3), Total HDL.

NMR lipoprotein particle size: VLDL size, LDL size, HDL size.

NMR lipoprotein particle levels (pno): Large VLDL, Medium VLDL, Small VLDL, Total VLDL (VLDLpno), Small LDL, Medium LDL, Total LDL, Large HDL (H4+H5pno), Medium HDL, Total HDL.

${ }^{\#}$ standardized odds ratio: $\operatorname{Exp}\left(\beta^{*}\right.$ standard deviation $)$

\section{Discussion}

This prospective study suggests that NMR-derived lipoprotein measures, ie. total VLDL particle number and large and medium size HDL subclasses predict CAD in Type 1 diabetic patients, independently of previously recognized risk factors, including renal disease which was also an independent predictor. Of particular interest is the positive association of medium HDL with CAD in contrast to the usual negative HDL association. It is difficult to make definite conclusions about which particular subclasses are independent predictors of CAD, due to multiple testing and small $p$ values, however, from univariate analyses all VLDL subclasses, small and medium LDL and medium and large HDL subclasses as well as average LDL and HDL size seem to be related to the development of CAD.

To date, studies in Type 1 diabetic patients with cardiovascular disease and enzymatically- measured lipids and lipoproteins show increased concentrations of total and LDL cholesterol and total and VLDL triglycerides in the cases $[4,7,8,40,41]$. The majority of these studies, however, did not adjust for other risk factors, and none have used NMR spectroscopy.

$V L D L$. In univariate analysis, all three NMR-derived VLDL subclasses were related to CAD, whether levels were expressed as particle or triglyceride mass concentrations. Enzymatically-measured triglycerides were also an important univariate predictor. In multivariate analysis, total VLDL particle number was an independent predictor of CAD. 
While there is little comparable data on VLDL subclasses, prior studies in Type 1 diabetes $[40,42]$ and in non-diabetic subjects (using the NMR technique) [43] support a relation between VLDL and cardiovascular disease. In contrast, smaller chylomicron and VLDL "remnant" particles and post prandial hyperlipidaemia have classically been viewed as atherogenic [44, 45]. Although increased levels of triglycerides have been correlated to other atherogenic and prothrombotic changes such as increases in small dense LDL and decreases in HDL cholesterol [13, 14], the availability of these latter variables in the current analyses suggests that the VLDL subclass particles are indeed of primary importance. This is further supported by a meta-analysis in the general population which showed that an increase in plasma triglycerides was associated with both a $32 \%$ increased cardiovascular risk in men and a $76 \%$ increased risk in women, independent of HDL cholesterol and other risk factors [46].

$L D L$. Total LDL cholesterol concentrations, measured enzymatically and calculated by Friedewald or estimated by NMR spectroscopy, were not associated with CAD. However, LDL particle concentrations measured by NMR or estimated by apolipoprotein B levels were strongly related to CAD. The explanation for this difference is that the small and medium LDL subclasses are the LDL fractions associated with CAD. Since small LDL particles contain considerably less cholesterol than larger LDL, they make a relatively small contribution to total LDL cholesterol mass than they do to the total LDL particle number. Thus, LDL particle concentration shows a stronger univariate disease association than LDL cholesterol. A probable reason for the failure of small, medium LDL and total LDL particle number to predict CAD independently in multivariate analyses is the high correlation of these variables with other lipids and lipoproteins, making it difficult to assess independence. In non-diabetic subjects, small LDL has been shown to be associated with up to a three-fold increased risk of coronary artery disease independent of age, sex and weight $[13,14]$. Our results also confirm previous findings of the EDC study (8-year follow-up), where small dense LDL (measured by ultracentrifugation) was associated with $\mathrm{CAD}$, but was only of borderline significance and statistically explained by triglycerides [47]. Similarly to LDL, no independent association was shown with IDL although the increased proportion of detectable IDL in those with CAD in univariate analysis could suggest that IDL might play a small role, in Type 1 diabetes.

$H D L$. HDL has been proposed to protect against the development of cardiovascular disease by facilitating transport of cholesterol from peripheral cells to the liver by reverse cholesterol transport [48]. For HDL subclasses, there is some confusion in the literature as to the nature and relative importance of different subclasses in terms of their effect on cardiovascular disease. Some studies observed a "protective" effect for large $\mathrm{HDL}$ subfractions $\left(\mathrm{HDL}_{2 \mathrm{~b}}\right)$ [15] while others focus on an increased risk of CVD for subjects with small HDL particles $\left(\mathrm{HDL}_{3 \mathrm{~b}}\right.$ and ${ }_{3 c}$ ) [16]. The current study suggests that increased medium HDL subclass could play an additional role in the prediction of CHD. Another cross-sectional study, dividing three LpA-I subclasses by gradient gel electrophoresis reported similar findings in normotriglyceridaemic and hypertriglyceridaemic CAD patients [49]. Inverse associations were found with CAD for the large and positive associations for the intermediate subclass [49]. Further study is needed to determine the mechanistic role of medium HDL (if any) in terms of cholesterol efflux. The solitary positive association between medium HDL and triglycerides suggests it could reflect disordered VLDL catabolism. There are a number of important clinical inferences from these data. These include recognition that as HDL cholesterol levels generally are higher in Type 1 diabetes, it would, given the current results, be unwise to attribute any benefit, thereby, as some of the increase is likely to reflect the possibly atherogenic medium HDL subclass. Furthermore, as previously suggested, these findings of clear differences in HDL subfractions in Type 1 diabetes should caution against using the same level to determine risk status as in the general population.

NMR method. We believe that NMR spectroscopy has improved our ability to characterize those Type 1 diabetic subjects at increased risk for CAD events. The ability to provide data on particle concentration clearly shows that the total number of VLDL particles is the critical measure. In the HDL subfractions, NMR clearly shows a subclass which in contrast to the general picture, shows a direct positive relationship to CAD risk. The value of NMR subclass measures is also shown in the LDL data, which clearly shows a risk for small and medium LDL subclass.

An important issue is whether to report NMR lipoprotein data in particle or lipid mass concentration terms. In our data, total LDL particle concentration is related more strongly to CAD than total LDL cholesterol mass, whereas total HDL particle concentration is slightly less strongly associated with CAD than total HDL cholesterol mass. These results are in accord with the observed strengths of CAD association with the small and medium LDL and large HDL subclasses, since on theoretical grounds it is expected that lipoprotein particle concentrations will be weighted more heavily by contributions made by smaller, relatively lipid-poor particles, whereas lipoprotein lipid mass concentrations will be weighted more heavily by contributions made by larger, lipid-rich particles. It 
would thus seem that both particle and lipid mass concentrations are of relevance.

These data also suggest that use of individual lipoprotein subclass levels might be more useful than overall mean particle sizes. Indeed, mean particle sizes did not improve the prediction of CAD compared to enzymatically-measured lipids and conventional risk factors. Consistent with our results, no clear relationship between particle size and calcification was shown in a recent publication on Type 1 diabetic patients [20].

EDC study design. The positive aspects of this study are its prospective design, focus on a sample of Type 1 diabetic patients, independently identified and relatively representative and the standardized assessment of cardiovascular endpoints. The main weakness was the relatively small study sample size. Nevertheless, there were several significant findings. The use of frozen plasma samples stored for 6 to 14 years for the NMR spectroscopy measurements represents a potential limitation of our study. However, data from other studies indicate negligible differences in lipoprotein particle sizes and subclass levels in fresh and frozen samples. Moreover, any theoretical random misclassification due to freezing artifacts would tend to bias our results towards the null. A potential limitation of the NMR spectroscopy method is that the conversion factors for expressing "particle" mass as "lipid" mass were derived from non-diabetic subjects. Another weakness of this study is that cases of angina were not validated by stress testing and similarly CAD could not be fully excluded in the control group, as full cardiac evaluation is not feasible in epidemiological studies.

Statin therapy. In this study, lipid lowering drugs were used by only one CAD case and none of the controls and had, therefore, little influence on the results. Similarly, there are no independent associations on CAD for other medication types including diuretics, beta-blockers, calcium-channel blockers, ACE inhibitors and aspirin. Recently, some trials have shown that statin therapy reduced NMR spectroscopy-measured lipoproteins, with reductions in VLDL subclasses and large LDL and increases in large HDL subclasses found in Type 2 diabetic patients with a modest dyslipidemia and prior CAD (Soedamah-Muthu et al. unpublished 2002) and reductions in small or large LDL subclasses depending on what subclass was most predominant at baseline in non-diabetic patients with prior CAD [50]. Whether statin therapy will have a beneficial impact on NMRmeasured lipoproteins, particularly medium HDL, in Type 1 diabetic patients remains to be established.

Total VLDL particle number and overt nephropathy were positive independent predictors of CAD in this population of Type 1 diabetic subjects, while levels of medium HDL were positively predictive and large HDL was negatively predictive. These results suggest that lipoprotein subclass measurements by NMR spectroscopy could further refine the lipoprotein prediction of CAD in Type 1 diabetes.

Acknowledgements. This study was supported by a National Institutes of Health Grant DK-34-818.

We would like to thank all study participants who volunteered their time, the staff of the Diabetes Research Center, and our numerous consultants.

\section{References}

1. Portuese E, Orchard T (1995) Mortality in insulin-dependent diabetes. In: Diabetes in America, pp 221-232

2. Dullaart RP (1995) Plasma lipoprotein abnormalities in type 1 (insulin-dependent) diabetes mellitus. Neth J Med 46:44-54

3. The DCCT Research Group (1992) Lipid and lipoprotein levels in patients with IDDM diabetes control and complication. Trial experience. The DCCT Research Group. Diabetes Care 15:886-894

4. Taskinen MR (1990) Hyperlipidaemia in diabetes. Baillieres Clin Endocrinol Metab 4:743-775

5. Lahdenpera S, Groop PH, Tilly KM et al (1994) LDL subclasses in IDDM patients: relation to diabetic nephropathy. Diabetologia 37:681-688

6. Jensen T, Stender S, Deckert T (1988) Abnormalities in plasmas concentrations of lipoproteins and fibrinogen in type 1 (insulin-dependent) diabetic patients with increased urinary albumin excretion. Diabetologia 31:142-145

7. Jensen T, Borch JK, Kofoed EA, Deckert T (1987) Coronary heart disease in young type 1 (insulin-dependent) diabetic patients with and without diabetic nephropathy: incidence and risk factors. Diabetologia 30:144-148

8. Laakso M, Pyorala K, Sarlund H, Voutilainen E (1986) Lipid and lipoprotein abnormalities associated with coronary heart disease in patients with insulin-dependent diabetes mellitus. Arteriosclerosis 6:679-684

9. Reckless JP, Betteridge DJ, Wu P, Payne B, Galton DJ (1978) High-density and low-density lipoproteins and prevalence of vascular disease in diabetes mellitus. Br Med J $1: 883-886$

10. Rivellese A, Riccardi G, Romano G et al. (1988) Presence of very low density lipoprotein compositional abnormalities in type 1 (insulin-dependent) diabetic patients; effects of blood glucose optimisation. Diabetologia 31:884-888

11. Bagdade JD, Subbaiah PV (1989) Whole-plasma and highdensity lipoprotein subfraction surface lipid composition in IDDM men. Diabetes 38:1226-1230

12. Lamarche B, Tchernof A, Moorjani S et al. (1997) Small, dense low-density lipoprotein particles as a predictor of the risk of ischemic heart disease in men. Prospective results from the Quebec Cardiovascular Study. Circulation 95:69-75

13. Gardner CD, Fortmann SP, Krauss RM (1996) Association of small low-density lipoprotein particles with the incidence of coronary artery disease in men and women. JAMA 276:875-881

14. Austin MA, Breslow JL, Hennekens CH et al. (1988) Lowdensity lipoprotein subclass patterns and risk of myocardial infarction. JAMA 260:1917-1921

15. Katzel LI, Coon PJ, Busby MJ et al. (1992) Reduced HDL2 cholesterol subspecies and elevated postheparin hepatic lipase activity in older men with abdominal obesity and asymptomatic myocardial ischemia. Arterioscler Thromb 12:814-823 
16. Cheung MC, Brown BG, Wolf AC, Albers JJ (1991) Altered particle size distribution of apolipoprotein A-I-containing lipoproteins in subjects with coronary artery disease. J Lipid Res 32:383-394

17. Otvos JD Measurement of Lipoprotein subclass profile by nuclear magnetic resonance spectroscopy. Clin Lab 48:171-180

18. Otvos JD, Jeyarajah EJ, Bennett DW, Krauss RM (1992) Development of a proton nuclear magnetic resonance spectroscopic method for determining plasma lipoprotein concentrations and subspecies distributions from a single, rapid measurement. Clin Chem 38:1632-1638

19. Lyons TJ, Zheng D, Otvos JD et al. (2000) Nuclear Magnetic Resonance (NMR)-Determined lipoprotein subclass profile in the DCCT/EDIC Cohort: associations with retinopathy and nephropathy. Diabetes 49:A269

20. Colhoun HM, Otvos JD, Rubens MB et al. (2002) Lipoprotein subclasses and particle sizes and their relationship with coronary artery calcification in men and women with and without type 1 diabetes. Diabetes 51:1949-1956

21. Orchard TJ, Dorman JS, Maser RE et al. (1990) Prevalence of complications in IDDM by sex and duration. Pittsburgh Epidemiology of Diabetes Complications Study II. Diabetes 39:1116-1124

22. Wagener DK, Sacks JM, LaPorte RE, Macgregor JM (1982) The Pittsburgh study of insulin-dependent diabetes mellitus. Risk for diabetes among relatives of IDDM. Diabetes 31:136-144

23. Hypertension detection and follow-up program cooperative group (1976) The hypertension detection and follow-up program: hypertension detection and follow-up program cooperative group. Prev Med 5:207-215

24. Bucolo G, David H (1973) Quantitative determination of serum triglycerides by the use of enzymes. Clin Chem 19:476-482

25. Allain CC, Poon LS, Chan CS, Richmond W, Fu PC (1974) Enzymatic determination of total serum cholesterol. Clin Chem 20:470-475

26. Warnick GR, Albers JJ (1978) A comprehensive evaluation of the heparin-manganese precipitation procedure for estimating high density lipoprotein cholesterol. J Lipid Res 19:65-76

27. Friedewald WT, Levy RI, Fredrickson DS (1972) Estimation of the concentration of low-density lipoprotein cholesterol in plasma, without use of the preparative ultracentrifuge. Clin Chem 18:499-502

28. Cruickshanks KJ, Orchard TJ, Becker DJ (1985) The cardiovascular risk profile of adolescents with insulindependent diabetes mellitus. Diabetes Care 8:118-124

29. Mendoza SG, Zerpa A, Carrasco H et al. (1983) Estradiol, testosterone, apolipoproteins, lipoprotein cholesterol, and lipolytic enzymes in men with premature myocardial infarction and angiographically assessed coronary occlusion. Artery 12:1-23

30. Williams KV, Erbey JR, Becker D, Arslanian S, Orchard TJ (2000) Can clinical factors estimate insulin resistance in type 1 diabetes? Diabetes 49:626-632

31. Otvos JD, Jeyarajah EJ, Bennett DW (1991) Quantification of plasma lipoproteins by proton nuclear magnetic resonance spectroscopy. Clin Chem 37:377-386

32. Redgrave TG, Carlson LA (1979) Changes in plasma very low density and low density lipoprotein content, composition, and size after a fatty meal in normo- and hypertriglyceridemic man. J Lipid Res 20:217-229

33. Grundy SM, Vega GL, Otvos JD, Rainwater DL, Cohen JC (1999) Hepatic lipase activity influences high density lipoprotein subclass distribution in normotriglyceridemic men.
Genetic and pharmacological evidence. J Lipid Res 40:229-234

34. Blake GJ, Otvos JD, Rifai N, Ridker PM (2002) Lowdensity lipoprotein particle concentration and size as determined by nuclear magnetic resonance spectroscopy as predictors of cardiovascular disease in women. Circulation 106:1930-37

35. Rumsey SC, Galeano NF, Arad Y, Deckelbaum RJ (1992) Cryopreservation with sucrose maintains normal physical and biological properties of human plasma low density lipoproteins. J Lipid Res 33:1551-1561

36. Klein R, Klein BE, Moss SE (1989) The Wisconsin epidemiological study of diabetic retinopathy: a review. Diabetes Metab Rev 5:559-570

37. Ellis D, Buffone GJ (1977) New approach to evaluation of proteinuric states. Clin Chem 23:666-670

38. Maser RE, Steenkiste AR, Dorman JS et al. (1989) Epidemiological correlates of diabetic neuropathy. Report from Pittsburgh Epidemiology of Diabetes Complications Study. Diabetes 38:1456-1461

39. The DCCT Research Group. (1988) Factors in development of diabetic neuropathy. Baseline analysis of neuropathy in feasibility phase of Diabetes Control and Complications Trial (DCCT). Diabetes 37:476-481

40. Koivisto VA, Stevens LK, Mattock M et al. (1996) Cardiovascular disease and its risk factors in IDDM in Europe. EURODIAB IDDM Complications Study Group. Diabetes Care 19:689-697

41. Fuller JH, Stevens LK, Wang SL (2001) Risk factors for cardiovascular mortality and morbidity: the WHO Mutinational Study of Vascular Disease in Diabetes. Diabetologia 44 [Suppl 2]:S54-S64

42. Forrest KY, Becker DJ, Kuller LH, Wolfson SK, Orchard TJ (2000) Are predictors of coronary heart disease and lower-extremity arterial disease in type 1 diabetes the same? A prospective study. Atherosclerosis 148:159-169

43. Freedman DS, Otvos JD, Jeyarajah EJ et al. (1998) Relation of lipoprotein subclasses as measured by proton nuclear magnetic resonance spectroscopy to coronary artery disease. Arterioscler Thromb Vasc Biol 18:1046-1053

44. Karpe F, Hellenius ML, Hamsten A (1999) Differences in postprandial concentrations of very-low-density lipoprotein and chylomicron remnants between normotriglyceridemic and hypertriglyceridemic men with and without coronary heart disease. Metabolism 48:301-307

45. Ebenbichler CF, Kirchmair R, Egger C, Patsch JR (1995) Postprandial state and atherosclerosis. Curr Opin Lipidol 6:286-290

46. Hokanson JE, Austin MA (1996) Plasma triglyceride level is a risk factor for cardiovascular disease independent of high-density lipoprotein cholesterol level: a meta-analysis of population-based prospective studies. J Cardiovasc Risk 3:213-219

47. Erbey JR, Robbins D, Forrest KY, Orchard TJ (1999) Lowdensity lipoprotein particle size and coronary artery disease in a childhood-onset type 1 diabetes population. Metabolism 48:531-534

48. Brewer HB (1999) Hypertriglyceridemia: changes in the plasma lipoproteins associated with an increased risk of cardiovascular disease. Am J Cardiol 83:3F-12F

49. Decossin C, Castro G, Derudas B et al. (1997) Subclasses of LpA-I in coronary artery disease: distribution and cholesterol efflux ability. Eur J Clin Invest 27:299-307

50. Otvos JD, Shalaurova I, Freedman DS, Rosenson RS (2002) Effects of pravastatin treatment on lipoprotein subclass profiles and particle size in the PLAC-I trial. Atherosclerosis 160:41-48 